\title{
Two Reaction Routes for the Preparation of Aromatic Polyoxadiazoles and Polytriazoles: Syntheses and Properties
}

\author{
E. R. HENSEMA, ${ }^{\star}$ J. P. BOOM, M. H. V. MULDER, ${ }^{\dagger}$ and C. A. SMOLDERS \\ University of Twente, Department of Chemical Technology, P.O. Box 217, 7500 AE Enschede, The Netherlands
}

\begin{abstract}
SYNOPSIS
Two reaction routes for the preparation of aromatic poly-1,3,4-oxadiazoles and poly-1,2,4triazoles are studied and their influence on the physical properties, i.e., inherent viscosity, glass transition, degradation temperature, and film integrity of the final products are discussed. Aromatic poly-1,3,4-oxadiazoles are prepared by means of a polycondensation reaction of terephthaloyl chloride and isophthalic dihydrazide yielding a precursor polymer, poly ( $p, m$-phenylene) hydrazide, which is converted into the corresponding poly-1,3,4-oxadiazole by means of a cyclodehydration reaction. Poly-1,3,4-oxadiazoles are also prepared by means of a polycondensation reaction between terephthalic and isophthalic acid and hydrazine yielding poly-1,3,4-oxadiazoles with higher inherent viscosities. Flexible poly1,3,4-oxadiazole films are obtained only if the inherent viscosities of the polymers used are higher than $2.7 \mathrm{dL} / \mathrm{g}$. The thermal stability is found to increase with increasing content of $p$-phenylene groups in the polymer backbone. Aromatic poly-1,2,4-triazoles are prepared using polyhydrazides with alternating para- and meta-phenylene groups and poly-1,3,4oxadiazoles with a random incorporation of para- and meta-phenylene groups in the main chain as precursor polymers. The glass transition temperatures are found to increase with increasing content of $p$-phenylene groups in the main chain of these polymers. Cold crystallization is observed only for the alternating polymer. (c) $1994 \mathrm{John}$ Wiley \& Sons, Inc.

Keywords: poly-1,3,4-oxadiazoles $\bullet$ polyhydrazide $\bullet$ poly-1,2,4-triazoles $\bullet$ cold crystallization
\end{abstract}

\section{INTRODUCTION}

Aromatic poly-1,3,4-oxadiazoles and poly-1,2,4triazoles are chemically resistant and thermally stable polymers soluble only in strong acids and stable up to $450^{\circ} \mathrm{C}$. ${ }^{1}$ We have studied these polymers as new membrane materials, ${ }^{2,3}$ with the aim to develop membranes that can be applied under severe operating conditions, such as elevated temperatures and acidic or basic environments. Such membranes also should be highly resistant towards organic solvents. A number of reaction routes are known for the preparation of these poly-1,3,4-oxadiazoles and poly1,2,4-triazoles. ${ }^{4}$

We decided to study the preparation of aromatic poly-1,3,4-oxadiazoles by means of a solid state cy-

* Present address: CIBA-GEIGY AG, Central Research Laboratories, CH-4002 Basel, Switzerland.

To whom correspondence should be addressed.

Journal of Polymer Science: Part A: Polymer Chemistry, Vol. 32, 513-525 (1994) (c) 1994 John Wiley \& Sons, Inc. $\mathrm{CCC} 0887-624 \mathrm{X} / 94 / 030513-13$ clodehydration reaction of an aromatic polyhydrazide, ${ }^{5,6}$ the latter being used as a tractable precursor polymer. Since poly-1,3,4-oxadiazoles with inferior mechanical properties were obtained, ${ }^{2}$ a second reaction route was also studied. This route directly yields aromatic poly-1,3,4-oxadiazoles from hydrazine sulphate, and terephthalic and isophthalic acid as monomers. ${ }^{7}$ Aromatic poly-1,2,4-triazoles were prepared by a reaction of an aromatic polyhydrazide ${ }^{8}$ and poly-1,3,4-oxadiazoles ${ }^{9}$ with aniline in polyphosphoric acid.

The synthesis routes are represented in Figure 1. Polyhydrazides with alternating para- and metaphenylene groups in the main chain were prepared using terephthaloyl chloride and isophthaloyl dihydrazide as monomers. Conversion of this precursor polymer yields poly-1,3,4-oxadiazoles and poly-1,2,4triazoles with alternating $p$ - and $m$-phenylene groups ( see reaction route $\mathrm{A}$ ).

Employing mixtures of terephthalic and isophthalic acid in different ratios in combination with 

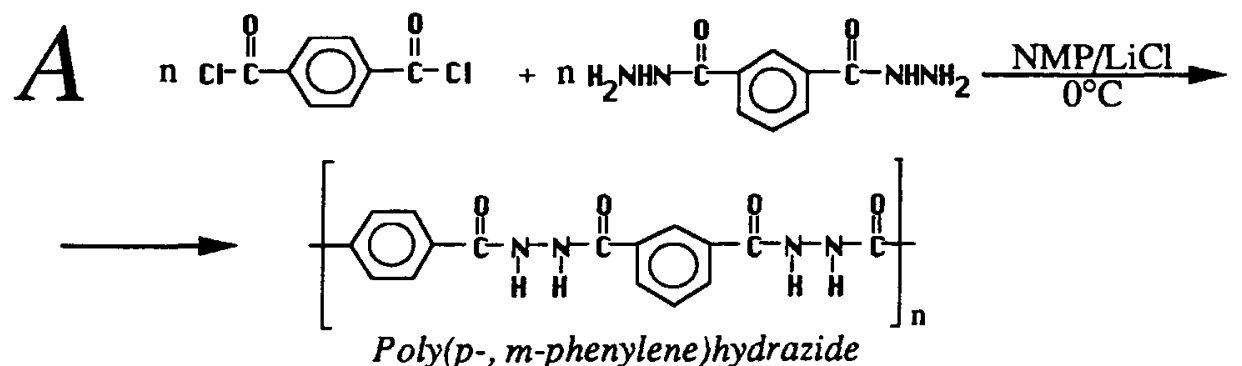

(I)

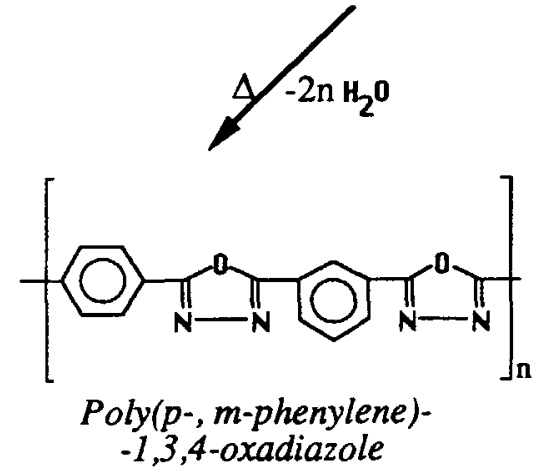

(II)
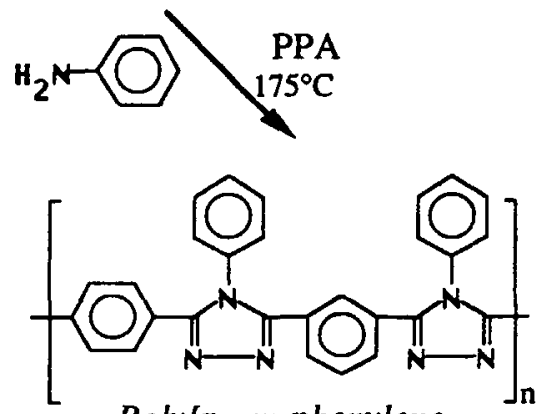

Poly[p-, m-phenylene-(4-phenyl)-1,2,4-triazole]

(III)
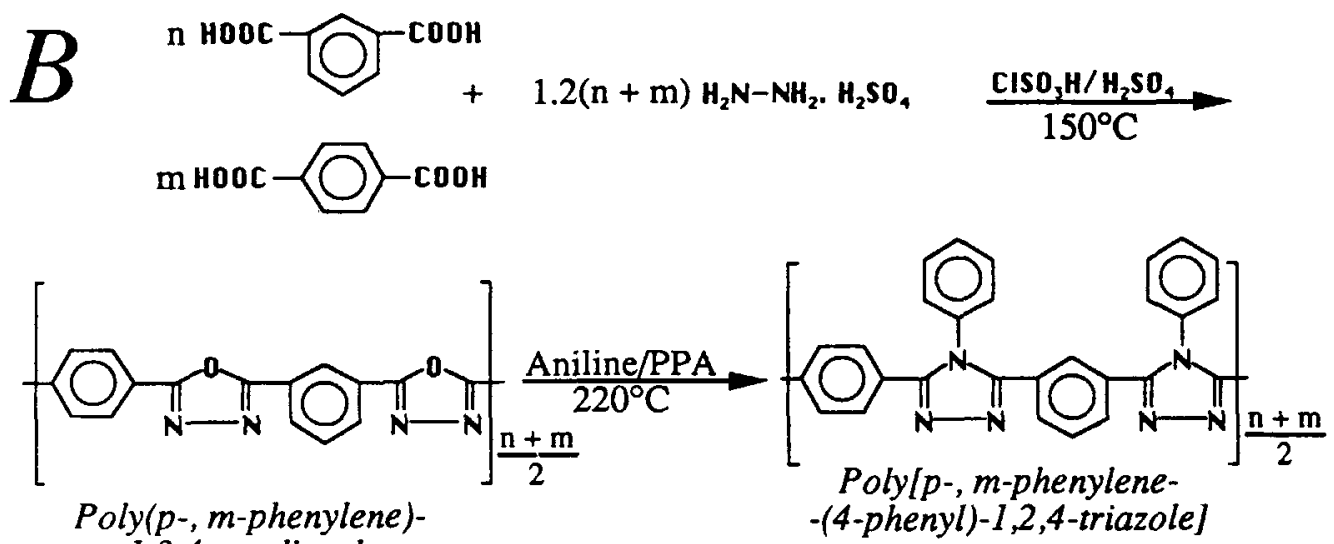

-I,3,4-oxadiazole

(IV)

(V)

\section{$P P A=$ PolyPhosphoric Acid}

Figure 1. (A) Preparation of alternating poly ( $p-, m$-phenylene)-1,3,4-oxadiazole and poly [ $p$-, $m$-phenylene- (4-phenyl) -1,2,4-triazole ] via poly ( $p$-, $m$-phenylene) -hydrazide. (B) Direct preparation of random poly ( $p-, m$-phenylene) $-1,3,4$-oxadiazoles and conversion into poly [ $p$-, $m$-phenylene- (4-phenyl) -1,2,4-triazoles $]$.

hydrazine sulphate results in the direct formation of poly-1,3,4-oxadiazoles where $p$ - and $m$-phenylene groups are incorporated randomly (see reaction route $B$ ). These poly-1,3,4-oxadiazoles were used as a precursor polymer for the poly-1,2,4-triazole syn- thesis. They were converted into poly-1,2,4-triazoles, having the same incorporation of $p$ - and $m$-phenylene groups in the main chain as the precursor polymer, by means of a reaction with aniline in polyphosphoric acid. 
In this article we wish to report the synthesis routes used and the properties of the polymers prepared. The gas separation properties of membranes of these polymers are discussed elsewhere. ${ }^{10}$

\section{EXPERIMENTAL}

\section{Materials}

All chemicals were supplied by Merck, and were used as received, except NMP, which was distilled before use under reduced pressure over $\mathrm{CaH}_{2}$, lithium chloride, which was dried in a vacuum oven at $150^{\circ} \mathrm{C}$, and aniline, which was distilled under reduced pressure. The colorless distillate was stored under nitrogen in a sealed flask in a refrigerator.

Isophthaloyl dihydrazide was prepared from isophthalic dimethyl ester and hydrated hydrazine hydrate. ${ }^{11}$

\section{Poly ( $p$-, m-phenylene $)$ hydrazide Synthesis (1 $)^{5,6}$}

Isophthaloyl dihydrazide, $74.242 \mathrm{~g}(0.382 \mathrm{~mol})$ and $16.21 \mathrm{~g} \mathrm{LiCl}$ are dissolved in a 3-necked flask containing $600 \mathrm{~mL}$ NMP at $60^{\circ} \mathrm{C}$ under dry nitrogen. After dissolution the stirred solution is cooled to $0^{\circ} \mathrm{C}$ and $77.619 \mathrm{~g}(0.382 \mathrm{~mol})$ terephthaloyl chloride is added in 4 equal portions. Each portion is added when the terephthaloyl chloride of the previous portion has completely dissolved. Total addition lasts approximately $2 \mathrm{~h}$. The viscous solution is stirred overnight, and the polymer is precipitated and washed in hyperfiltrated demineralized water. Subsequently the polymer is washed in ethanol and dried in a vacuum oven at $150^{\circ} \mathrm{C}$ for at least $24 \mathrm{~h}$.

\section{Poly-1,3,4-oxadiazole Synthesis (II) Using (I) as a Precursor Polymer $2,5,6$}

Alternating poly ( $p$-, $m$-phenylene) -1,3,4-oxadiazole is prepared by heating solid poly $(p-, m$-phenylene) hydrazide in a nitrogen atmosphere. Various heating cycles have been applied, including: $24 \mathrm{~h}$ at $276^{\circ} \mathrm{C}, 24 \mathrm{~h}$ at $288^{\circ} \mathrm{C}, 24 \mathrm{~h}$ at $285^{\circ} \mathrm{C}, 62 \mathrm{~h}$ at $280^{\circ} \mathrm{C}$, followed by $100 \mathrm{~h}$ at $300^{\circ} \mathrm{C}$ and a heating cycle of $48 \mathrm{~h}$ at $310^{\circ} \mathrm{C}$.

\section{Poly-1,2,4-triazole Synthesis (III) Using (I) as Precursor Polymer $^{8}$}

In a 3-necked flask $310 \mathrm{~g}$ polyphosphoric acid (PPA) is heated to $150^{\circ} \mathrm{C}$ under nitrogen. Aniline, $76.65 \mathrm{~g}$ $(0.823 \mathrm{~mol})$ is added dropwise to the stirred solution, meanwhile maintaining the temperature at $150^{\circ} \mathrm{C}$. After addition, the temperature is raised to $175^{\circ} \mathrm{C}$, and $10 \mathrm{~g}$ poly ( $p-, m$-phenylene $)$ hydrazide is added. The reaction mixture is kept at this temperature for $140 \mathrm{~h}$. This mixture is poured into hyperfiltrated demineralized water and neutralized with sodium hydroxide under stirring. The product is washed in a $5 \%$ sodium hydroxide solution and twice in hyperfiltrated demineralized water. Subsequently, the product is extracted with ethanol using a Soxhlet extractor, after which it is dried in a vacuum oven at $150^{\circ} \mathrm{C}$ for $24 \mathrm{~h}$. The product is dissolved in formic acid, and insoluble parts are removed by means of filtration over a $5 \mu \mathrm{m}$ filter. Homogeneous poly-1,2,4triazole films are directly prepared from these solutions, as discussed below.

\section{One-Step Synthesis of Poly-1,3,4-oxadiazoles (IV) ${ }^{12}$}

To $18.75 \mathrm{~g}(0.14 \mathrm{~mol})$ hydrazine sulphate, in a 3 necked flask and in a dry nitrogen atmosphere, is added $230 \mathrm{~mL}$ chlorosulfonic acid. To this stirred solution is added $30 \mathrm{~mL} 30 \% \mathrm{SO}_{3}$ fuming sulphuric acid and $140 \mathrm{~mL}$ concentrated sulphuric acid, followed by the addition of $20 \mathrm{~g}(0.12 \mathrm{~mol})$ of a mixture of terephthalic and isophthalic acid and $1.2 \mathrm{~g}$ benzene sulphonic acid. (Hydrazine is employed in a 20 mol \% excess yielding poly-1,3,4-oxadiazoles with higher inherent viscosities than obtained under equimolar conditions. $)^{7}$ After addition, the temperature is raised to $135^{\circ} \mathrm{C}$ and stirred at this temperature until the viscosity of the reaction mixture has reached its maximum lasting $2 \mathrm{~h}$ for poly ( $p$-phenylene )-1,3,4-oxadiazole and $8 \mathrm{~h}$ for poly ( $m$-phenylene ) -1,3,4-oxadiazole.

The reaction mixture is cautiously poured into ice water and washed with hyperfiltrated demineralized water for $24 \mathrm{~h}$. The polymer is dried at $150^{\circ} \mathrm{C}$ in a vacuum oven for at least $24 \mathrm{~h}$.

Poly ( $p$-phenylene)-1,3,4-oxadiazole was also prepared using only fuming sulphuric acid as a reaction medium. ${ }^{7}$ After addition of the reactants, the temperature is raised to $90^{\circ} \mathrm{C}$ and maintained at this temperature for $2 \mathrm{~h}$. Subsequently, the temperature is raised to $130^{\circ} \mathrm{C}$ and kept at this temperature for another $5 \mathrm{~h}$. After completion, the reaction mixture is poured into hyperfiltrated demineralized water. Further purification is identical to the procedure described above.

\section{Poly-1,2,4-triazole Synthesis (V) Using (IV) as a Precursor Polymer ${ }^{9}$}

Random poly ( $p$-, $m$-phenylene) -1,3,4-oxadiazoles were used as precursor polymers for the preparation of the corresponding poly-1,2,4-triazoles. The same 
procedure as described for the preparation of poly1,2,4-triazoles using polyhydrazide as a precursor polymer is applied, however the reaction temperature is, in this case, $225^{\circ} \mathrm{C}$, while a reaction time of $35 \mathrm{~h}$ is required. ${ }^{9}$ This procedure, described by D'Yachenko et al., yielded poly-1,2,4-triazoles after reaction times much longer than $35 \mathrm{~h}$ and in yields of only $5-10 \%$, will be discussed below. For this reason the, synthesis was altered; first, the poly-1,3,4oxadiazole is dissolved in PPA at $150^{\circ} \mathrm{C}$ followed by the addition of aniline. The temperature is raised to $225^{\circ} \mathrm{C}$ and maintained at this temperature. After $35 \mathrm{~h}$ the reaction mixture is poured into hyperfiltrated demineralized water and the precipitate is purified as described under "Poly-1,2,4-triazole synthesis (III) using (I) as precursor polymer."

\section{Preparation of Homogeneous Films}

\section{Poly (p-, m-phenylene) hydrazide Films}

Homogeneous transparent colorless polyhydrazide films are prepared by casting a $10-15$ wt $\%$ polymer solution in DMSO onto a glass plate using a casting knife with a slit height of $0.30 \mathrm{~mm}$, followed by evaporation of the solvent at $80^{\circ} \mathrm{C}$ under a continuous nitrogen flow. The film is removed from the glass plate and dried at $150^{\circ} \mathrm{C}$ in a vacuum oven to remove residual solvent. Films are obtained with thicknesses of $20-60 \mu \mathrm{m}$.

\section{Poly (p-, m-phenylene-(4-phenyl)-1,2,4-triazole] Films}

Homogeneous poly-1,2,4-triazole films are prepared by casting a $15 \mathrm{wt} \%$ polymer solution in formic acid on a glass plate using casting knives with slit heights of 0.3 and $0.5 \mathrm{~mm}$, followed by evaporation of the solvent in a nitrogen atmosphere at room temperature. After evaporation, the glass plate is immersed into a water bath and the poly-1,2,4-triazole film is easily removed from the glass plate. Transparent yellow films are obtained with thicknesses of 20-60 $\mu \mathrm{m}$.

\section{Poly (p-, m-phenylene)-1,3,4-oxadiazole Film}

Poly-1,3,4-oxadiazole films are obtained by solid state cyclodehydration of polyhydrazide films, obtained as described above, using different heating cycles and heating times.

Poly-1,3,4-oxadiazole films were also obtained by casting a 4-5 wt \% polymer solution in concentrated sulphuric acid onto a glass plate using a casting knife with a slit height of $0.5 \mathrm{~mm}$, followed by immersion in a bath containing a $50 \mathrm{wt} \%$ aqueous sulphuric acid solution. The film is rinsed with demineralized water during $24 \mathrm{~h}$ followed by immersion into an ethanol bath and a hexane bath. The films are dried between polysulphone micro-filtration membranes under slight pressure to prevent curling. The transparent poly-1,3,4-oxadiazole film is removed from the polysulphone membranes with the help of a little water and dried in a vacuum oven at $70^{\circ} \mathrm{C}$. The thickness of these films was approximately $50 \mu \mathrm{m}$.

Thirdly, poly-1,3,4-oxadiazole films are obtained by casting the sulphuric acid diluted reaction mixture of poly( $p$-phenylene)-1,3,4-oxadiazole acid onto a glass plate. The reaction mixture is diluted with sulphuric acid in order to reduce the viscosity of this casting solution. The solution is cast on a glass plate followed by immersion into a $50 \mathrm{wt} \%$ aqueous sulphuric acid solution. The transparent film is rinsed with demineralized water and dried in a vacuum oven.

\section{Characterization}

\section{Inherent Viscosity}

The inherent viscosities are determined of $0.5 \mathrm{~g}$ polymer solutions in $100 \mathrm{~mL}$ solvent at $30^{\circ} \mathrm{C}$. For the polyhydrazides DMSO, for the poly-1,2,4-triazoles formic acid, and for the poly-1,3,4-oxadiazoles, concentrated sulphuric acid is used as a solvent.

\section{Differential Scanning Calorimetry (DSC)}

DSC measurements are performed on a Perkin-Elmer DSC 4 in combination with a System 4 Microprocessor Controller and a Thermal Analysis Data Station (TADS), model 3700. The polymer samples are placed in aluminum sample pans, and the temperature is increased with a heating rate of $20^{\circ} \mathrm{C} /$ min from 50 to $450^{\circ} \mathrm{C}$ under a nitrogen purge gas stream. As glass transition temperature, the midpoint of the enthalpy change is taken, calculated by means of the TADS software.

\section{Thermogravimetrical Analysis (TGA)}

TGA experiments are carried out on a Perkin-Elmer TGS-2 in combination with a System 4 Microprocessor Controller and a Thermal Analysis Data Station, model 3700. Experiments are carried out under a nitrogen-purge gas stream from $50-600^{\circ} \mathrm{C}$. The temperature of the polymer samples is increased with a heating rate of $20^{\circ} \mathrm{C} / \mathrm{min}$ and the weight loss over a certain temperature interval is monitored. 
Table I. Elemental Analysis of Poly( $p$-, $m$-phenylene)hydrazide and Poly( $p$-, $m$-phenylene)1,3,4-oxidiazoles

\begin{tabular}{|c|c|c|c|c|c|c|}
\hline $\begin{array}{c}\text { Polymer } p / m \\
\text { Ratio }\end{array}$ & $\begin{array}{c}\eta_{\text {inh }}{ }^{a} \\
(\mathrm{dI} / \mathrm{g})\end{array}$ & $\begin{array}{c}\mathrm{C} \\
(\mathrm{wt} \%)\end{array}$ & $\begin{array}{c}\mathrm{N} \\
\text { (wt \%) }\end{array}$ & $\begin{array}{c}\mathrm{H} \\
\text { (wt \%) }\end{array}$ & $\begin{array}{c}\mathrm{C} / \mathrm{N} \\
(\mathrm{wt} \% / \mathrm{wt} \%)\end{array}$ & \\
\hline PH-p/m-1 & Alternating & $0.82^{\mathrm{b}}$ & 56.05 & 16.02 & 3.58 & 3.50 \\
\hline POD-p/m-1 & Alternating & & 65.39 & 19.21 & 2.77 & 3.40 \\
\hline POD-0/100-1 & $0 / 100$ & 1.5 & 66.15 & 19.58 & 2.92 & 3.38 \\
\hline POD-25/75-1 & $25 / 75$ & 1.9 & 66.06 & 19.20 & 2.94 & 3.44 \\
\hline POD-25/75-2 & $25 / 75$ & 1.2 & 62.70 & 18.43 & 2.76 & 3.44 \\
\hline POD-33/67-1 & $33 / 67$ & 2.0 & 66.30 & 19.72 & 2.88 & 3.36 \\
\hline POD-50/50-1 & $50 / 50$ & 2.7 & 64.55 & 18.78 & 2.85 & 3.44 \\
\hline POD-50/50-2 & $50 / 50$ & 1.6 & 63.33 & 18.04 & 2.47 & 3.51 \\
\hline POD-50/50-3 & $50 / 50$ & 4.2 & 64.22 & 18.43 & 2.91 & 3.48 \\
\hline POD-67/33-1 & $67 / 33$ & 2.5 & 64.72 & 19.02 & 2.80 & 3.40 \\
\hline POD-75/25-1 & $75 / 25$ & 2.0 & 66.06 & 19.23 & 2.85 & 3.44 \\
\hline POD-75/25-2 & $75 / 25$ & 1.4 & 63.74 & 18.54 & 2.86 & 3.44 \\
\hline POD-75/25-3 & $75 / 25$ & 2.9 & 64.51 & 19.11 & 2.80 & 3.38 \\
\hline POD-100/0-1 & $100 / 0$ & 0.9 & 63.42 & 19.08 & 2.92 & 3.32 \\
\hline POD-100/0-2 & $100 / 0$ & 2.2 & 59.20 & 16.60 & 2.89 & 3.56 \\
\hline \multirow[t]{2}{*}{ Theoretical values } & $\mathrm{PH}$ & & 59.26 & 17.28 & 3.73 & 3.43 \\
\hline & POD & & 66.67 & 19.44 & 2.80 & 3.43 \\
\hline
\end{tabular}

anherent viscosity determined in concentrated sulphuric acid.

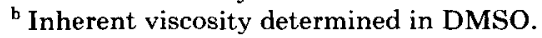

\section{Thermomechanical Measurements}

A Myrenne torsion pendulum is used to obtain thermomechanical spectra of the poly-1,2,4-triazoles from -175 to $350^{\circ} \mathrm{C}$. The temperature of the samples is increased with a heating rate of $1.8^{\circ} \mathrm{C} / \mathrm{min}$. The applied frequency was $1 \mathrm{~Hz}$. Poly-1,2,4-triazole coated glass fibers are used as samples. For this purpose glass fibers were heated at $450^{\circ} \mathrm{C}$ during several hours to remove any coating. Subsequently, a bundle of fibers was dip-coated in a poly-1,2,4-triazole solution in formic acid followed by evaporation of the solvent. Residual solvent was removed at $70^{\circ} \mathrm{C}$ in a vacuum oven. Samples with dimensions of $55 \times 1$ $\times 0.75 \mathrm{~mm}$ are obtained.

\section{Wide-Angle X-Ray Diffractograms (WAXS)}

WAXS diffractograms are recorded on a Philips Röntgen diffractometer, PW 1710 using copper diffraction $(\mathrm{Cu} \mathrm{K} \alpha, \lambda=1.5418 \AA)$.

\section{RESULTS AND DISCUSSION}

\section{Poly-1,3,4-oxadiazoles via Polyhydrazides}

A number of polyhydrazides were prepared and their IR spectra are in agreement with the IR spectrum reported by Frazer et al. ${ }^{6}$ The results of the elemental analysis of one of the polyhydrazides, $\mathrm{PH}-\mathrm{p} / \mathrm{m}-1$, is represented in Table I. The experimental values found are somewhat lower than the theoretical ones. Nonetheless, the $\mathrm{C} / \mathrm{N}$ quotient is close to its theoretical value indicating that the polyhydrazide contains residual impurities.

Polyhydrazides with inherent viscosities up to $1.39 \mathrm{dL} / \mathrm{g}$ were obtained (see Table II). From all these polymers, flexible transparent films were prepared. Polyhydrazides having higher inherent viscosities are reported when hexamethyl phosphor-

Table II. Inherent Viscosities of Poly $(p-$, $m$-phenylene)hydrazides, as Prepared, before, and after Thermal Conversion ${ }^{2}$

\begin{tabular}{cllc}
\hline & \multicolumn{2}{c}{ Conversion Cycles } & \\
\cline { 2 - 3 } $\begin{array}{c}\text { Polyhydrazide } \\
\eta_{\text {inh }}(\mathrm{dL} / \mathrm{g})^{\mathrm{a}}\end{array}$ & Time $(\mathrm{h})$ & $\mathrm{T}\left({ }^{\circ} \mathrm{C}\right)$ & $\begin{array}{c}\text { Polyoxadiazole } \\
\eta_{\text {inh }}(\mathrm{dL} / \mathrm{g})^{\mathrm{b}}\end{array}$ \\
\hline 0.69 & 24 & 276 & 0.30 \\
0.69 & 24 & 288 & 0.33 \\
1.07 & $62 / 100$ & $280 / 300$ & 0.55 \\
1.17 & $62 / 100$ & $280 / 300$ & 0.64 \\
1.39 & 48 & 310 & 0.86 \\
\hline
\end{tabular}

anherent viscosity determined in DMSO.

${ }^{\mathrm{b}}$ Inherent viscosity determined in concentrated sulphuric acid. 
amide, HMPA, is used as a solvent instead of NMP. ${ }^{8}$ HMPA was not used since it is very carcinogenic. ${ }^{13}$

Films of polyhydrazide $\mathrm{PH}-\mathrm{p} / \mathrm{m}-1$, see Table I, were converted into poly-1,3,4-oxadiazole films by heating the films during $24 \mathrm{~h}$ at $285^{\circ} \mathrm{C}$ in a nitrogen atmosphere. The values of elemental analysis of the converted polyhydrazide, $\mathrm{POD}-\mathrm{p} / \mathrm{m}-1$, are in agreement with the theoretical values ( see Table I). The IR-spectrum is similar to the one reported by Frazer et al. $^{6}$ where the same characteristic absorption peaks are found $(=\mathrm{C}-\mathrm{O}-\mathrm{C}=$ stretching at 960 $\mathrm{cm}^{-1}$ and $>\mathrm{C}=\mathrm{N}-$ stretching at $1550 \mathrm{~cm}^{-1}$ ).

Various polyhydrazide films were isothermally converted into poly-1,3,4-oxadiazole films in a nitrogen atmosphere in a furnace at temperatures between 250 and $320^{\circ} \mathrm{C}$ for $24-100 \mathrm{~h}$. Conversion resulted in all cases in shrunken and embrittled poly1,3,4-oxadiazole films. ${ }^{2}$ The films were so brittle that they disintegrated at the slightest force applied to them. No mechanical properties could be measured.

Embrittlement of converted polyhydrazide films has also been reported by Frazer and Sarasohn. ${ }^{14}$ This is in contrast to reported flexible converted polyhydrazide fibers prepared by the same group. ${ }^{15,16}$ In both cases, amorphous polyhydrazides with similar inherent viscosities were obtained. Cumbersome conversions of polyhydrazide-related polyamidehydrazides into poly-1,3,4-oxadiazole-amides resulting in the inferior mechanical qualities of the latter are also reported by Preston et al. ${ }^{17}$

The observed brittleness is likely to be caused by chain shrinkage upon conversion, a low molecular weight, a lack of orientation, or, possibly, a combination of these phenomena.

Macroscopic shrinkage is caused by shrinkage of polyhydrazide macromolecules upon conversion into poly-1,3,4-oxadiazoles. Shrinkage of these macromolecules is caused by the ring closure of the hydrazide groups into the 1,3,4-oxadiazole groups. $\mathrm{X}$ ray analysis showed that upon ring closure, the carbon-carbon distance in hydrazide segment decreases by $30 \% .^{17,18}$ This means for the presently investigated poly ( $p$-, $m$-phenylene ) -hydrazide, a size reduction of approximately $16 \%$ per monomeric unit upon conversion. If this were a freely jointed chain, its end-to-end distance would also have decreased by $16 \%$, since the end-to-end distance is proportional to the unit length. A polyhydrazide film does not, however, consist of freely jointed chains. The glassy poly ( $p-, m$-phenylene) hydrazide matrix consists of entangled polymers stiffening and shrinking upon cyclodehydration into the corresponding poly-1,3,4oxadiazole, which results in stress build-up in the polymer matrix that contributes to the embrittlement of the film.

Polyhydrazide films were observed to undergo a dimensional decrease of approximately $12 \%$ during a thermal conversion of $48 \mathrm{~h}$ at $300^{\circ} \mathrm{C}$, slightly deviating from the calculated $16 \%$, obviously due to hindered shrinkage in the solid state.

Conversion of oriented poly ( $p-, m$-phenylene) hydrazide fibers yielded poly-1,3,4-oxadiazole fibers with superior mechanical properties. ${ }^{20} \mathrm{We}$ speculate that this orientation results in a controlled contraction of the polyhydrazide fiber upon cyclodehydration and, thus, minimizing stress build-up, this in contrast to the nonoriented polyhydrazide film.

The observed brittleness cannot be explained in terms of stress build-up alone, since dissolving converted polyhydrazide in sulphuric acid, and thus eliminating stresses, followed by casting of this polymer solution onto a glass plate and immersion into a $50 \mathrm{wt} \%$ aqueous sulphuric acid bath did not yield a flexible film. From this experiment it can be concluded that another factor is responsible for the observed brittleness. Besides stress build-up, mechanical behavior of the converted films is, of course, determined by the molecular weight of the poly1,3,4-oxadiazole formed in situ, which is obviously too low, since no flexible film is obtained after the above described immersion casting process. A similar strong dependence between intrinsic viscosity and the mechanical properties of converted poly ( $p-, m$ phenylene) hydrazide fibers is also reported by Frazer and Reed. ${ }^{20}$ Polyhydrazide films are flexible because of strong intermolecular forces due to hydrogen bonding between hydrazide groups in the polyhydrazide macromolecules. During a heating cycle, these strongly interacting and flexible hydrazide groups are converted into rigid and less interacting 1,3,4-oxadiazole groups. A certain minimal intermolecular adherence between the macromolecules is required to attain flexibility of the final product. It is, therefore, believed that higher molecular weight poly-1,3,4-oxadiazoles are needed to overcome this decline in macromolecular interactions and thus maintain flexibility of films and fibers. Low molecular weight of the final poly-1,3,4-oxadiazole can be due to the fact that the molecular weight of the initial polyhydrazide is too low, or may be caused by a decrease in molecular weight due to chain rupture during conversion. These two phenomena may, of course, also occur simultaneously.

The inherent viscosities of poly-1,3,4-oxadiazoles obtained applying different polyhydrazides and 
heating cycles are represented in Table II. Poly1,3,4-oxadiazoles with inherent viscosities varying between 0.30 to $0.86 \mathrm{dL} / \mathrm{g}$ were obtained. ${ }^{2}$ It is shown that there exists a proportional relationship between the inherent viscosities of polyhydrazides and poly-1,3,4-oxadiazoles. The inherent viscosity is obviously only a function of the inherent viscosity of the precursor polymer and is not influenced by the temperature or the duration of the thermal conversion. One would expect a decreasing inherent viscosity with conversion time and applied temperature in the case of degradation.

It can thus be concluded that flexible poly-1,3,4oxadiazole films can only be obtained if polyhydrazides with higher inherent viscosities are used as a precursor polymer. However, stress build-up and chain shrinkage may then still have a deteriorating effect on the mechanical properties of poly-1,3,4oxadiazole films.

\section{One-Step Synthesis of Poly-1,3,4-oxadiazoles}

Poly-1,3,4-oxadiazoles with higher inherent viscosities are easily prepared by a one-step synthesis using dicarboxylic acids and hydrazine as monomers. High molecular weight poly ( $p$-phenylene)-1,3,4-oxadiazoles were prepared using the reaction originally reported by Iwakura et al. ${ }^{7}$ Poly ( $p$-phenylene) 1,3,4-oxadiazoles having inherent viscosities from 2.9 to $3.2 \mathrm{dL} / \mathrm{g}$ were obtained. Flexible yellow films were obtained by casting the sulphuric acid diluted reaction mixture onto a glass plate followed by immersion into a $50 \mathrm{wt} \%$ aqueous sulphuric acid bath. Since low molecular weight poly-1,3,4-oxadiazoles were obtained when mixtures of terephthalic and isophthalic acid were used, another reaction procedure was chosen, where, instead of fuming sulphuric acid, a mixture of chlorosulphonic acid and $100 \%$ sulphuric acid was employed. ${ }^{12}$ Less colored products with moderate inherent viscosities were obtained, in all cases containing less than 0.7 wt \% sulphur. Poly-1,3,4-oxadiazoles with various ratios of para- and meta-phenylene groups using mixtures of terephthalic and isophthalic acid were obtained in nearly quantitative yield.

Elemental analysis indicates that the desired products were obtained (see Table I). The values found are somewhat lower than the theoretical values indicating some residual impurities. The quotient $\mathrm{C} / \mathrm{N}$ is in good agreement with the theoretical value. Iwakura removes residual impurities by boiling the products in water during $5 \mathrm{~h}$ prior to elemental analysis. ${ }^{7}$ In our case, further purification was omitted since these polymers are used as precursors for the poly-1,2,4-triazole synthesis.

Infrared spectra of poly-1,3,4-oxadiazoles prepared by cyclodehydration of polyhydrazides and directly by a reaction of dicarboxylic acids with hydrazine sulphate are identical and are in agreement with the spectra given by Frazer et al. ${ }^{6}$ and Iwakura et al. ${ }^{7}$

No or negligible sulphonation has taken place, since only minor quantities of sulphur are found in the final product, in all cases less than 0.25 wt \%, except for POD-25/75-2 and POD-100/0-1, both containing $0.7 \mathrm{wt} \%$ sulphur. Another argument against sulphonation is the fact that no enhancement in solubility is observed, a phenomenon often encountered upon sulphonation. The poly-1,3,4-oxadiazoles prepared are only soluble in concentrated sulphuric acid and in polyphosphoric acid. During polymerization, sulphonation must compete with the polycondensation reaction. Since only minor quantities of sulphur are found, the latter reaction takes place preferentially over the sulphonation reaction. Sulphonation might then additionally take place when the polycondensation reaction is complete. However, aryl-substituted 1,3,4-oxadiazoles are considered as chemically resistant materials; for example 2,5-diphenyl-1,3,4-oxadiazole is not effected by concentrated $\mathrm{HNO}_{3}$ or by a treatment with fuming $\mathrm{H}_{2} \mathrm{SO}_{4}$ at $100^{\circ} \mathrm{C}$ for $4 \mathrm{~h}^{21}$ It is, therefore, believed that residual sulphur is not bound to the polymer by means of a sulphonation reaction, but that it is present as $\mathrm{H}_{2} \mathrm{SO}_{4}$.

The polycondensation reaction is assumed to take place in two steps; first, a condensation reaction between hydrazine and dicarboxylic acid, followed by cyclodehydration of the hydrazide group into the 1,3,4-oxadiazole group, ${ }^{22}$ (see Fig. 2).

In the case of an incomplete reaction, unclosed hydrazide groups are present in the final product, and a carbonyl peak at $1720 \mathrm{~cm}^{-1}$ is observed in the IR-spectrum. The percentage of unclosed rings in

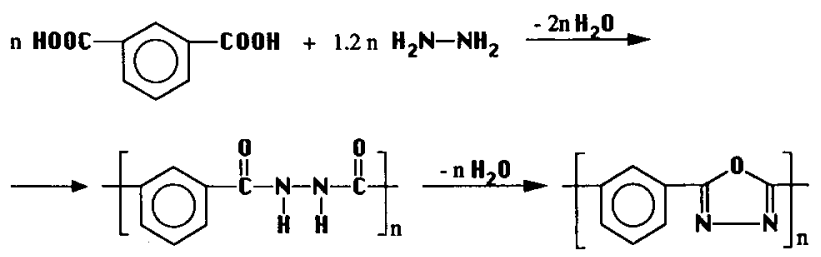

Figure 2. Two-step reaction mechanism for the formation of random poly ( $p-, m$-phenylene)-1,3,4-oxadiazoles using hydrazine sulphate and isophthalic acid as monomers. 


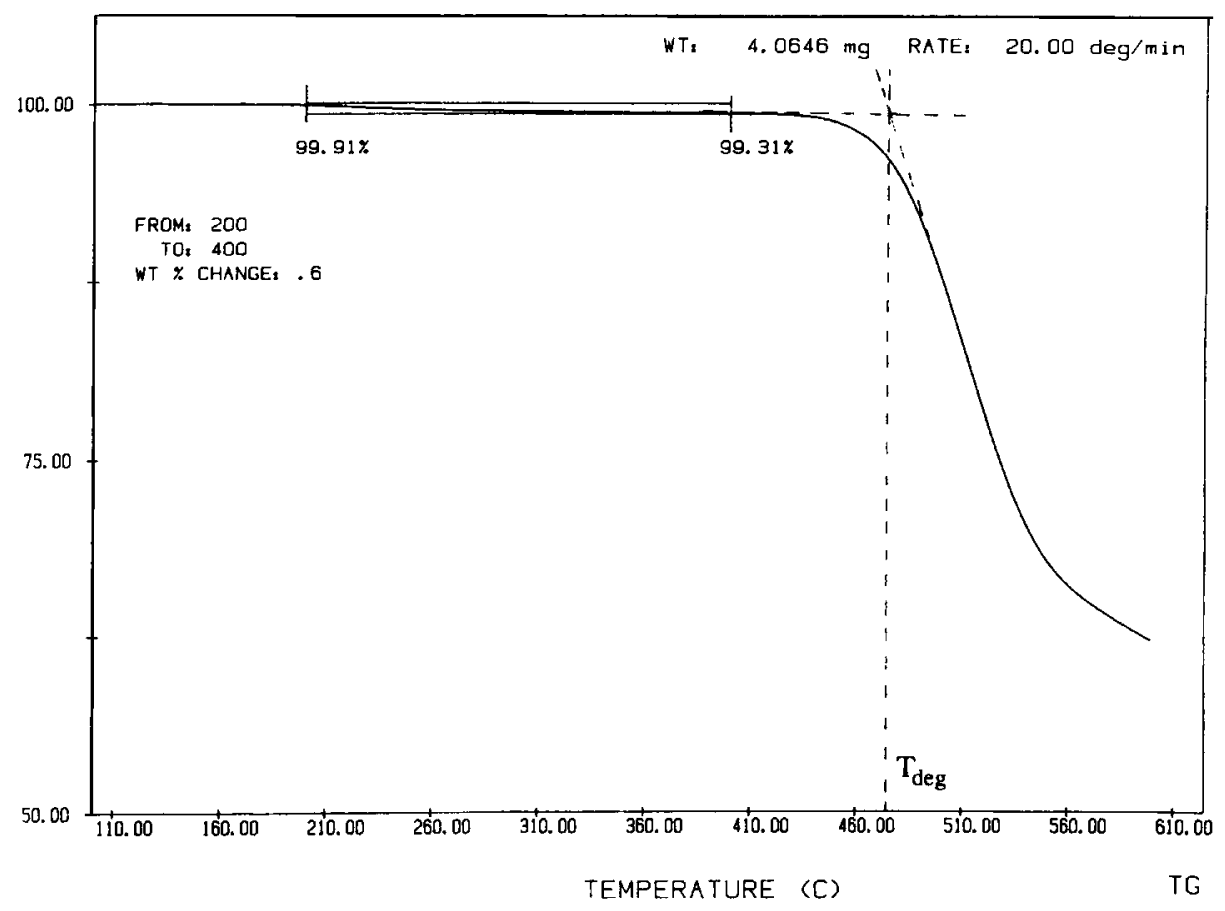

Figure 3. Thermogravimetrical weight loss curve POD-25/75-1 recorded at a heating rate of $20^{\circ} \mathrm{C} / \mathrm{min}$.

the final product can be determined by means of thermogravimetrical analysis. A TGA curve of POD75/25-3 is represented in Figure 3. Three distinct phases of weight loss can be observed: ${ }^{14}$ at approximately $100^{\circ} \mathrm{C}$ the loss of absorbed water, between 250 and $400^{\circ} \mathrm{C}$ a weight loss can be observed due to the loss of water caused by cyclodehydration of unclosed hydrazide groups into 1,3,4-oxadiazole groups. A maximal weight loss is observed here in the theoretical case when no ring has yet been closed at all. Such a thermogram is obtained for a poly $(p-, m$ phenylene) hydrazide where a maximal weight loss of $11.1 \%$ is recorded. From the actual weight loss curve, the percentage of unclosed rings can be calculated. A weight loss of $1.0 \%$ was observed for POD100/0-1 and POD-100/0-2, while for POD-25/752 a weight loss of $0.6 \%$ was recorded indicating the presence of 8 and $5 \%$ unclosed hydrazide groups, respectively. In all other cases, weight losses of less than $0.4 \%$ were observed until degradation. At temperatures above $450^{\circ} \mathrm{C}$, a weight loss is observed due to loss of volatiles indicating final degradation of the polymer.

The degradation temperature $T_{\operatorname{deg}}$ is defined as the point intersection of the tangents (see Fig. 3). In Figure 4, the degradation temperatures of the poly ( $p-, m$-phenylene ) -1,3,4-oxadiazoles are plotted versus the content of $p$-phenylene groups in the main chain. Increasing the content of $p$-phenylene results in a more thermally stable product likely due to resonance stabilization of the $p$-phenylene ring. These findings are in agreement with the results of Varma et al., ${ }^{23}$ who studied the thermal stability of poly ( $p$-, phenylene ) -1,3,4-oxadiazole and poly ( $m$ phenylene) -1,3,4-oxadiazole.

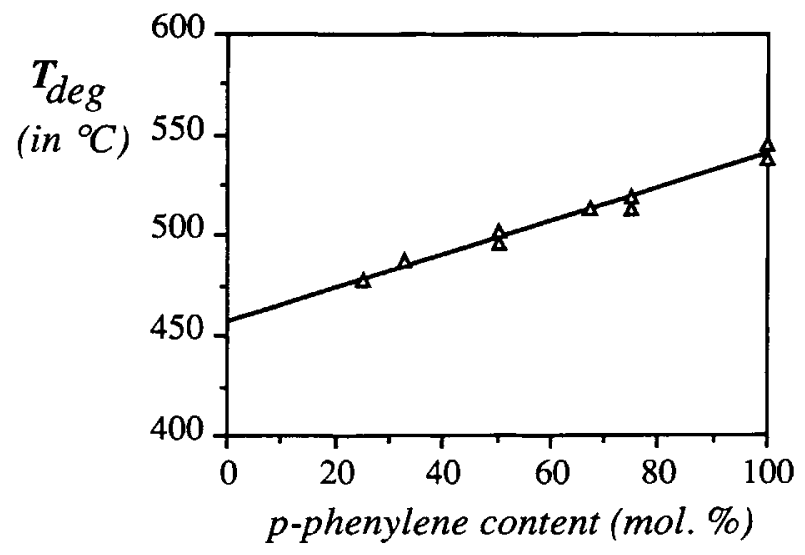

Figure 4. Degradation temperatures $\left(T_{\mathrm{deg}}\right)$ of random poly ( $p-, m$-phenylene) -1,3,4-oxadiazoles as a function of the amount of $p$-phenylene groups in the main chain. 
Table III. Elemental Analysis of Poly-1,2,4-triazoles

\begin{tabular}{|c|c|c|c|c|c|c|}
\hline Polymer & $p / m$-ratio & $\begin{array}{c}\eta_{\text {inh }}^{a} \\
(\mathrm{dL} / \mathrm{g})\end{array}$ & $\begin{array}{c}\mathrm{C} \\
(w t \%)\end{array}$ & $\begin{array}{c}\mathrm{N} \\
\text { (wt } \% \text { ) }\end{array}$ & $\begin{array}{c}\mathrm{H} \\
\text { (wt \%) }\end{array}$ & $\mathrm{C} / \mathrm{N}$ \\
\hline $\mathrm{PT}-p / m-1$ & Alternating & 0.85 & 73.04 & 18.02 & 4.06 & 4.05 \\
\hline PT-0/100-1 & $0 / 100$ & 0.73 & 71.73 & 17.51 & 3.87 & 4.10 \\
\hline $\mathrm{PT}-25 / 75-1$ & $25 / 75$ & 1.36 & 73.02 & 17.77 & 4.07 & 4.11 \\
\hline PT-50/50-1 & $50 / 50$ & 1.37 & 71.14 & 17.22 & 3.78 & 4.13 \\
\hline PT-75/25-1 & $75 / 25$ & 1.84 & 71.51 & 17.56 & 3.99 & 4.07 \\
\hline РТ-75/25-2 & $75 / 25$ & 1.60 & 73.5 & 18.2 & 4.1 & 3.99 \\
\hline Theoretical & & & 76.70 & 19.17 & 4.14 & 4.00 \\
\hline
\end{tabular}

${ }^{a}$ Inherent viscosity determined in formic acid.

No glass transition temperature was observed for the poly ( $p$-, $m$-phenylene) -1,3,4-oxadiazoles using DSC techniques, since these polymers undergo degradation before the glass transition is reached. ${ }^{24}$

Flexible films could be prepared from poly ( $p$ phenylene) -1,3,4-oxadiazoles prepared in fuming sulphuric acid with inherent viscosities of $2.8,2.9$, 3.0 , and $3.2 \mathrm{dL} / \mathrm{g}$, from POD-75/25-2 ( $\eta_{\mathrm{inh}}=2.9$ $\mathrm{dL} / \mathrm{g})$, from POD-50/50-1 $\left(\eta_{\mathrm{inh}}=2.7 \mathrm{dL} / \mathrm{g}\right)$, and from POD-50 $/ 50-3\left(\eta_{\mathrm{inh}}=4.2 \mathrm{dL} / \mathrm{g}\right)$. All other films disintegrated upon handling. Flexible poly-1,3,4oxadiazole films were investigated regarding their gas separation properties. ${ }^{10}$ Flexibility did not seem to depend on the ratio of $p / m$-phenylene groups in the main chain but merely on the inherent viscosity. A similar result is observed by Imai in the case of fibers; he obtained tough filaments only for random poly ( $p$-, $m$-phenylene) -1,3,4-oxadiazoles containing $75 \% p$ - and $25 \% m$-phenylene groups if the inherent viscosity was $1.7 \mathrm{dL} / \mathrm{g}$ or higher. ${ }^{25}$

$\mathrm{X}$-ray diffractograms of alternating poly $(p-, m$ phenylene) hydrazide powder indicate a completely amorphous product. In addition, diffractograms of POD-0/100-1, POD-50/50-3, and POD-100/0-1 are similar to the alternating polymer and even POD100/0-1, despite its more regular molecular structure, has almost no increased ordering as indicated by the identical WAXS diffractogram.

\section{Poly-1,2,4-triazole via Polyhydrazide}

The conversion reaction of poly $(p-, m$-phenylene)hydrazide into poly $[p-, m$-phenylene-(4phenyl) -1,2,4-triazole] was first investigated by Holsten and Lilyquist, ${ }^{8}$ who determined the ideal reaction conditions. Poly $[p-, m$-phenylene- (4phenyl) -1,2,4-triazoles ] with the highest inherent viscosities were obtained if the reaction was carried out at $175^{\circ} \mathrm{C}$ for $140 \mathrm{~h}$. Shorter reaction times are possible when higher reaction temperatures are employed but result in polymers with decreased inherent viscosities.

The elemental analysis of poly [ $p-, m$-phenylene(4-phenyl) -1,2,4-triazole ] PT-p/m-1 is given in Table III. The experimental values found are somewhat lower than the theoretical ones. The carbonyl absorption peak in the IR spectrum present in the precursor polymer has completely disappeared, while an absorption peak at $700 \mathrm{~cm}^{-1}$ due to the phenyl group has appeared in the spectrum of the poly-1,2,4triazole. The infrared spectrum is in agreement with the one of Korshak. ${ }^{26}$

A DSC thermogram of PT-p/m-1 is represented in Figure 5. PT-p $/ \mathrm{m}-1$ has a glass transition temperature of $264^{\circ} \mathrm{C}$. The exothermic peak at $319^{\circ} \mathrm{C}$ was identified as cold crystallization. ${ }^{27}$ WAXS experiments indicated that films of alternating poly[ $p$-, $m$-phenylene-(4-phenyl) -1,2,4-triazole] are completely amorphous. ${ }^{27}$

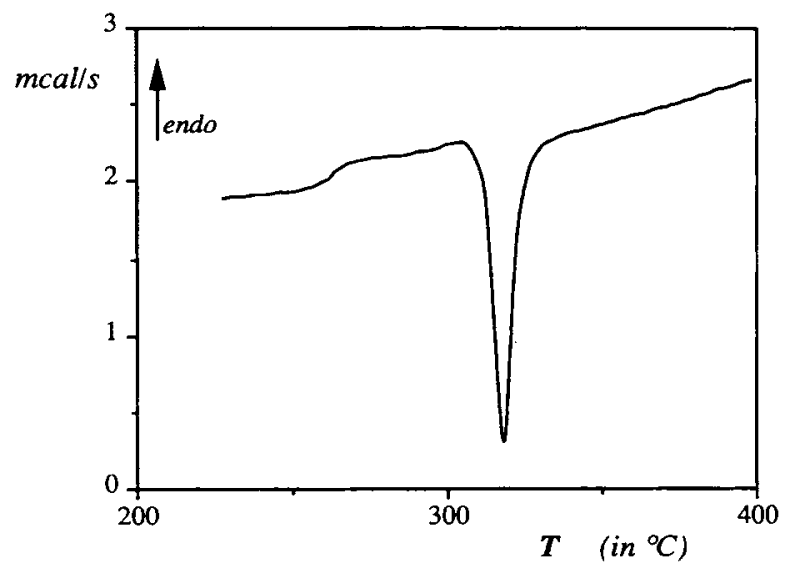

Figure 5. DSC thermogram for alternating poly $[p-, m$ phenylene- (4-phenyl)-1,2,4-triazole] (PT-p/m-1) recorded with a heating rate of $20^{\circ} \mathrm{C} / \mathrm{min}$ under nitrogen. 


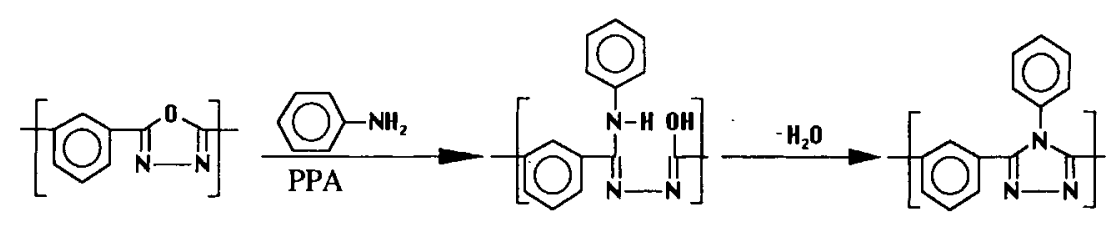

Figure 6. Formation of a poly (4-phenyl) -1,2,4-triazole out of a poly-1,3,4-oxadiazole via an intermediate.

\section{Poly-1,2,4-triazoles via Poly-1,3,4-oxadiazoles}

As precursor polymers, high molecular weight poly1,3,4-oxadiazoles are more easily obtained than polyhydrazides. While the polyhydrazide synthesis requires high-purity monomers and necessitates vigorous exclusion of water, making this synthesis a laborious one, the direct formation of poly-1,3,4oxadiazoles from dicarboxylic acids and hydrazine sulphate is relatively simple.

The conversion of poly ( $m$-phenylene ) -1,3,4-oxadiazoles and poly ( $p$-phenylene) -1,3,4-oxadiazoles into the corresponding poly-1,2,4-triazoles was first investigated by D'Yachenko et al., ${ }^{9}$ and the preparation is essentially the same as the method described by Holsten and Lilyquist where polyhydrazide is used as a precursor polymer. The reaction differs in its higher reaction temperature, $225^{\circ} \mathrm{C}$ in stead of $175^{\circ} \mathrm{C}$, and its shorter reaction time, $35 \mathrm{~h}$ instead of $140 \mathrm{~h}$. In both cases, the precursor polymer should dissolve during the reaction. In our case, the poly-1,3,4-oxadiazoles dissolved only to some extent during the reported $35 \mathrm{~h}$. During prolonged reaction times, up to $140 \mathrm{~h}$, the precursor poly-1,3,4-oxadiazoles dissolved completely but gave poly-1,2,4-triazoles in only a $5-10 \%$ yield. D'Yachenko et al. do not report slow dissolution of poly (phenylene) 1,3,4-oxadiazole in PPA. Their poly-1,3,4-oxadiazoles may have dissolved readily due to the low molecular weight of their precursor polymers.

We have modified the reaction procedure, and added the as-prepared poly-1,3,4-oxadiazoles to PPA prior to the addition of aniline.

Due to the addition of aniline to the PPA, the "solvent strength" of the latter is decreased. First, dissolving poly-1,3,4-oxadiazole in PPA followed by the addition of aniline is dissuaded, because of the expected degradation of the precursor polymer and, thus, the resulting low molecular weight poly-1,2,4triazoles. To show this effect, POD-25/75-2 was dissolved in PPA at $150^{\circ} \mathrm{C}$ and the inherent viscosity was determined as a function of time. Degradation takes place indeed, and the value of the inherent viscosity decreases by $20 \%$ during the first $15 \mathrm{~h}$ and remains constant thereafter until the end of the experiment. The decrease in inherent viscosity is, however, acceptable, and all the poly-1,2,4-triazoles represented in Table III were prepared by dissolving the precursor polymer in PPA followed by the addition of aniline.

The infrared spectra of these poly-1,2,4-triazoles are identical to the infrared spectrum of the poly1,2,4-triazole prepared via the polyhydrazide route. The elemental analysis indicates some impurities. Analysis showed residual amounts of phosphorous. Poly-1,2,4-triazoles with moderate inherent viscosities yielding films with good mechanical strength were obtained. These membranes were tested for their gas separation properties. ${ }^{10}$

The synthesis of poly-1,2,4-triazoles can further be simplified by performing the complete reaction as a one-pot synthesis. ${ }^{28}$ First the reaction between dicarboxylic acid and hydrazine yielding a poly-1,3,4oxadiazole is carried out in PPA. When this polycondensation reaction is complete, extra PPA is added to the reaction mixture followed by the addition of aniline. The reaction temperature is increased and the poly-1,3,4-oxadiazole is converted into the corresponding poly-1,2,4-triazole.

The poly-1,2,4-triazoles were characterized with DSC and TGA techniques. TGA experiments show the occurrence of a weight loss between $250^{\circ} \mathrm{C}$ and $400^{\circ} \mathrm{C}$ due to the formation and escape of water caused by a ring closure reaction of unclosed $1,2,4$ triazole groups. The reaction between poly-1,3,4oxadiazole and aniline is believed to occur via an intermediate $^{18}$ (see Fig. 6).

If the reaction is not complete, unclosed $1,2,4$ triazole rings are present in the final product. These groups will undergo cyclodehydration during a TGA experiment and the percentage of unclosed groups can be calculated (see Table IV).

These unclosed rings can be closed by means of a heat treatment at $350^{\circ} \mathrm{C}$ for $24 \mathrm{~h}$. Further investigations for the ideal reaction conditions should minimize the amount of unclosed rings, because they represent possible weak spots for thermally or chemically induced degradation. An increase in 
Table IV. TGA Analysis of Poly-1,2,4-triazoles

\begin{tabular}{lccc}
\hline & & $\begin{array}{c}\text { Relative } \\
\text { Weight Loss } \\
\text { (wt \%) }\end{array}$ & $\begin{array}{c}\text { Percentage } \\
\text { Unclosed } \\
\text { Rings } \\
(\%)\end{array}$ \\
\hline PT-p/m-1 & Alternating & 2.07 & 26 \\
PT-0/100-1 & $0 / 100$ & 0.88 & 11 \\
PT-25/75-1 & $25 / 75$ & 1.35 & 17 \\
PT-50/50-1 & $50 / 50$ & 2.47 & 31 \\
PT-75/25-1 & $75 / 25$ & 0.97 & 12 \\
PT-75/25-2 & $75 / 25$ & 1.30 & 16 \\
\hline
\end{tabular}

thermal stability with increasing ratio of paraphenylene groups in the main chain, as found for the poly-1,3,4-oxadiazoles, is not found for the poly$1,2,4$-triazoles. This is probably due to the presence of weak spots in the macromolecules, caused by prolonged exposure to the harsh reaction medium, PPA. These weak spots decrease the degradation temperature and overshadow the stability dependence on the ratio of para-/ meta-phenylene groups.

The TGA curve of the alternating poly $[p-, m-$ phenylene-(4-phenyl )-1,2,4-triazole] is similar to the curves of the random polymers with respect to degradation and ring closure behavior. The glass transition temperature increases with increasing $p$ phenylene ratio, as was shown by DSC and torsion pendulum experiments (see Fig. 7). This is due to an increased chain stiffness of the latter, which is also found by other authors for other copolymers. ${ }^{29,30}$ Upon extrapolation, a theoretical glass transition temperature of $380^{\circ} \mathrm{C}$ is obtained for poly [ $p$-phenylene-(4-phenyl)-1,2,4-triazole ]. A meaningful estimation of the glass transition temperature of poly [ $p$-phenylene- (4-phenyl) -1,2,4-triazole] using the Woods ${ }^{31}$ or Gordon-Taylor ${ }^{32}$ equation was not possible since too few glass transition temperatures of intermediate copolymers are available.

The glass transition temperature of the alternating poly $[p-, \quad m$-phenylene-(4-phenyl)-1,2,4-triazoles] is slightly lower than the $T_{g}$ of the random variant, possibly due to the higher molecular weight of the latter.

When the $p$-phenylene content was $75 \%$, no glass transition temperature was detectable with DSC. With a torsion pendulum, the glass transition was readily detected. In the DSC thermogram, the magnitude of the heat capacity jump at the glass transition is proportional to the amorphous fraction of the polymer. ${ }^{33}$ Increasing the ratio of $p$-phenylene groups gives a more linear polymer, which obviously results in a polymer matrix with increased ordering and a decreased amorphous fraction. In Figure 8, WAXS diffractograms indicate that this is indeed the case.

The exothermic peak representing cold crystallization in the rubbery phase is completely absent in the case of random poly $[p-, m$-phenylene- (4phenyl)-1,2,4-triazoles] including the $100 \%$ metaphenylene variant. Alternating and random poly [ $p-$, $m$-phenylene- (4-phenyl) -1,2,4-triazoles] films are prepared similarly out of formic acid solutions. If cold crystallization were to occur in the case of only completely amorphous poly $[p-, m$-phenylene- (4phenyl )-1,2,4-triazoles] then PT-0/100-1 and PT25/75-1 should also undergo cold crystallization, since they are also completely amorphous as indicated by WAXS diffractograms (see Fig. 8). It is believed that cold crystallization can only occur for poly-1,2,4-triazoles where para- and meta-phenylene groups are incorporated alternatingly. Similar subtle influences of the macromolecular structure on cold crystallization have also been reported by Burfield and Tanaka. ${ }^{34}$ They found that cold crystallization in the rubbery phase occurred much more readily in the case of natural rubber, which is a completely cis1,4-polyisoprene product, than in the case of synthetic analogues of $99 \%$ cis $-1,4$ purity. The cold crystallization phenomenon is obviously very sensitive to macromolecular irregularities. In the alternating poly-1,2,4-triazole, para- and meta-phenylene groups are incorporated in a perfectly alternatingly fashion and are capable of achieving a crystalline

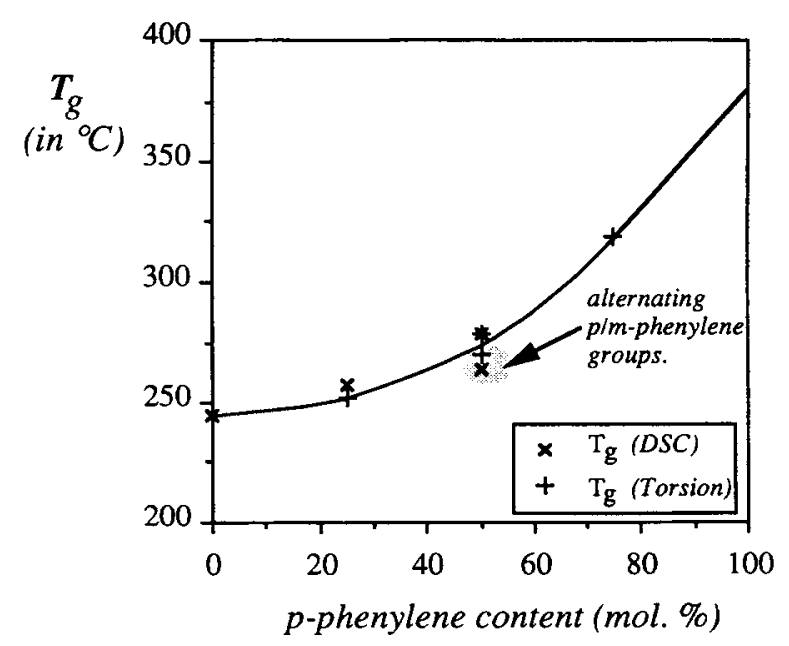

Figure 7. Glass transition temperatures $\left(T_{g}\right)$ of poly $[p-$ , $m$-phenylene- (4-phenyl)-1,2,4-triazoles] as a function of the ratio of $p$-phenylene groups in the main chain. 


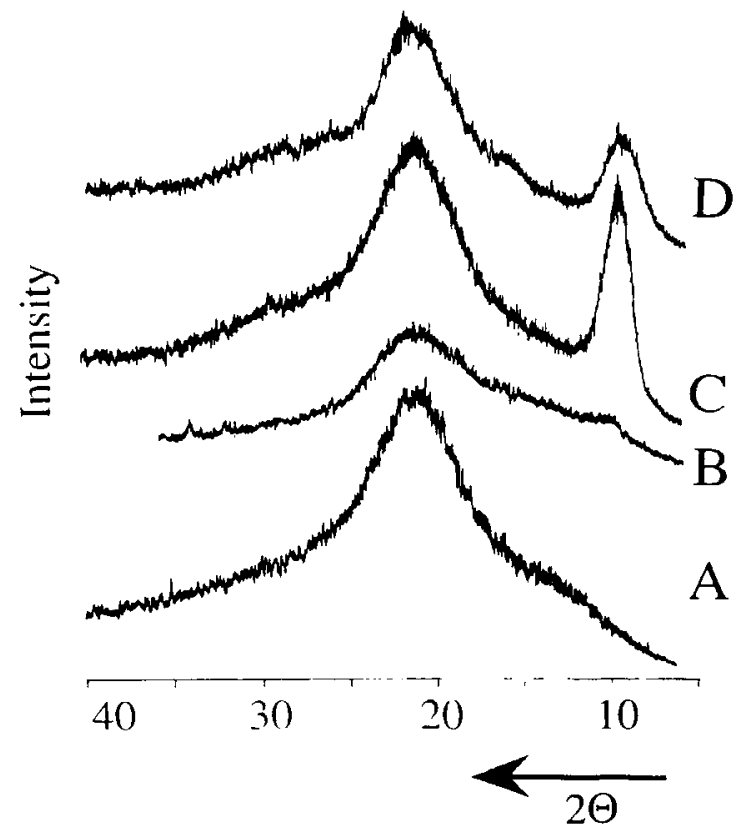

Figure 8. X-ray diffractograms for (A) PT-0/100-1, (B) PT-25/75-1, (C) PT-50/50-1, and (D) PT-75/25-1.

morphology upon cold crystallization, whereas, in the case of the random poly-1,2,4-triazoles, the $p$-/ $m$-phenylene groups are incorporated randomly and obviously lack the possibility of increasing their crystallinity through cold crystallization.

\section{CONCLUSIONS}

Aromatic poly-1,3,4-oxadiazoles are prepared by means of a polycondensation reaction of terephthaloyl chloride and isophthalic dihydrazide and by means of a polycondensation reaction between terephthalic and isophthalic acid and hydrazine sulphate. The latter reaction is more simple and yields poly-1,3,4-oxadiazoles with higher inherent viscosities. Flexible poly-1,3,4-oxadiazole films can be obtained only if the inherent viscosities of the polymers used are higher than $2.7 \mathrm{dL} / \mathrm{g}$. Thermal stability was found to increase with increasing content of $p$ phenylene groups.

Aromatic poly [ $p$-, $m$-phenylene (4-phenyl) -1,2,4triazoles ] were prepared using polyhydrazides with alternating para- and meta-phenylene groups and poly-1,3,4-oxadiazoles with a random incorporation of para- and meta-phenylene groups in the main chain as precursor polymers. Poly-1,2,4-triazoles were obtained in high yield after alteration of the original synthesis procedure given by D'Yachenko et al. ${ }^{9}$ The glass transition temperature was found to increase with increasing the ratio of $p$-phenylene groups in the main chain. Cold crystallization was only observed for the alternating polymer, and its occurrence is believed to be directly related to the manner of incorporation of the phenylene groups in the main chain.

The authors are indebted to T. Ploeger and J. E. G. J. Wijnhoven for carrying out a large number of syntheses and numerous experiments. Akzo Research is gratefully acknowledged for the financial support of this research project.

\section{REFERENCES AND NOTES}

1. P. E. Cassidy, Thermally stable polymers, Marcel Dekker, New York, 1980.

2. B. Gebben, Thermally stable and chemically resistant polymer membranes, Aromatic polyoxadiazoles and polytriazoles, $\mathrm{Ph}$. D. Thesis, University of Twente, The Netherlands, 1988.

3. B. Gebben, M. H. V. Mulder, and C. A. Smolders, J. Membrane Sci., 46, 29-41 (1989).

4. R. J. Cotter and M. Matzner, in A. T. Blomquist and H. Wasserman (Eds.), Organic Chemistry, Vol. 13B, 1, Academic Press, New York, 1972.

5. A. H. Frazer and F. T. Wallenberger, J. Polym. Sci., A2, 1147-1156 (1964).

6. A. H. Frazer, W. Sweeny, and F. T. Wallenberger, $J$. Polym. Sci., A2, 1157-1169 (1964).

7. Y. Iwakura, K. Uno, and S. Hara, J. Polym. Sci., A3, 45-54 (1965).

8. J. R. Holsten and M. R. Lilyquist, J. Polym. Sci., A3, 3905-3917 (1965).

9. A. V. D'Yachenko, V. V. Korshak, and E. S. Krongauz, Vysokomol. Soedin., Ser. A, 9(10), 2231-2235 (1967).

10. E. R. Hensema, J. P. Boom, M. E. R. Sena, M. H. V. Mulder, and C. A. Smolders, Polymer, to appear.

11. A. H. Frazer and F. T. Wallenberger, J. Polym. Sci., A2, 1137-1145 (1964).

12. Fr. Demande, 2.111 .913 (1972).

13. J. A. Zapp, Science, 190, 422 (1975).

14. A. H. Frazer and I. M. Sarasohn, J. Polym. Sei., Part $A-1,4,1649-1664$ (1966).

15. A. H. Frazer and D. R. Wilson, Applied Polymer Symposia, 9, 89-106 (1969).

16. A. H. Frazer and F. T. Wallenberger, J. Polym. Sci., Part A, 2, 1171-1179 (1964).

17. J. Preston, W. B. Black, and W. L. Hofferbert, J. Macromol. Sci.-Chem, A7, 45-65 (1973).

18. V. V. Korshak, G. L. Berestneva, I. P. Bragina, G. V. Yeremina, and V. V. Krylova, Vysokomol. Soyed., A16, 1714-1722 (1974).

19. V. V. Korshak, G. L. Berestneva, I. P. Bragina, and 
S. A. Astafiev, J. Polym. Sci., Polym. Symp., 47, 2534 (1974).

20. A. H. Frazer and T. A. Reed, J. Polym. Sci., C19, 89 94 (1967).

21. A. Hetzheim and K. Möckel, in A. R. Katritzky and A. J. Boulton (Eds.), Advances in heterocyclic chemistry, Vol. 7, Academic Press, New York, 1966.

22. V. V. Korshak, G. L. Berestneva, A. V. Volokhina, A. B. Raskina, D. V. Bibileishvili, P. V. Petrovskii, S. A. Astaf'ev, N. P. Okromchedlidze, and G. I. Kudryavtsev, Vysokomol. Soyed, A24, 36-42 (1982).

23. I. K. Varma, R. M. Sambadam, and D. S. Varma, Makromol. Chem., 170, 117-130 (1973).

24. B. Gebben, M. H. V. Mulder, and C. A. Smolders, $J$. Polym. Sci., Polym. Chem. Ed, 26, 1757-1768 (1988).

25. Y. Imai, J. Appl. Polym. Sci., 14, 225-239 (1970).

26. V. V. Korshak, E. S. Krongauz, and A. V. D'Yachenko, Doklady Akademii Nauk SSSR, 176, 338-340 (1967).
27. B. Gebben, M. H. V. Mulder, and C. A. Smolders, J. Polym. Sci., Polym. Chem. Ed, 27, 3481-3499 (1989).

28. A. H. Schroeder, P. Denisevich, S. Suzuki, and V. P. Kurkov, U.S. Pat. 4,519,940 (1985).

29. H. G. Weijland, P. J. Hoftijzer, and D. W. van Krevelen, Polymer, 11, 79-87 (1970).

30. D. R. Wiff, M. S. Altieri, and I. J. Goldfarb, J. Polym. Sci., Polym. Phys. Ed., 23, 1165-1176 (1985).

31. L. A. Woods, J. Polym. Sci., 38, 319-350 (1958).

32. M. Gordon and J. S. Taylor, J. Appl. Chem., 2, 493500 (1952).

33. J. Menczel and B. Wunderlich, Polym. Prepr., 1, 255256 ( 1986 ).

34. D. R. Burfield and Y. Tanaka, Polymer, 28, 907-910 (1987).

Received October 29, 1991

Accepted August 3, 1993 